\title{
Near-infrared imaging of the star forming region S87
}

\author{
Yuxi Chen ${ }^{1}$, Xing-Wu Zheng ${ }^{1}$, Yongqiang Yao $^{2}$, Ji Yang ${ }^{2}$, and Shuji Sato ${ }^{3}$ \\ 1 Department of Astronomy, Nanjing University, Nanjing 210093, PR China \\ 2 Purple Mountain Observatory, Chinese Academy of Science, 2 Beijing Xi Lu, Nanjing 210008, PR China \\ ${ }^{3}$ Department of Astrophysics, School of Science, Nagoya University, Chikusa-ku, Nagoya 464-01, Japan
}

Received 26 November 2001 / Accepted 26 August 2002

\begin{abstract}
Near-infrared images of the star forming region S87 in the $J H K^{\prime}$ broad-band filters and $\mathrm{H}_{2} v=1-0 \mathrm{~S}(1)$ narrow-band filter are presented. In the eastern part of the region, the S87E nebula shows a quite complex structure. In the optically thin southern part of the nebula, there is an elongated cavity in the direction aligned with the blue-shifted molecular outflow. A subcluster of twelve young near-infrared sources has been detected in the optically thick northern part of the nebula, where a cone-shaped opaque patch is seen. In the $90^{\prime \prime} \times 90^{\prime \prime}$ region around S87E, we detected 111 sources with $m_{K^{\prime}}>15.2$ mag. From analysis of the $J H K^{\prime}$ colors of the stars inside S87E, we find that after adjusting for background contamination, more than $40 \%$ of all the sources associated with the cluster display infrared excesses. This high fraction of infrared excess sources, along with the evidence of molecular outflow activity near the cluster, suggests that the S87E cluster is very young $\left(\sim 3-4 \times 10^{6}\right.$ yr $)$. We construct the differential $K^{\prime}$ luminosity function for S87E and find the overall shape and structure are somewhat different to that of other young clusters. Besides a common turnover at $14 \mathrm{mag}$, there is a prominent peak and still more cluster members with luminosities fainter than 14th magnitude at $K^{\prime}$ band. Five $\mathrm{H}_{2}$ regions were also discovered and three of them are associated with the S87E nebula.
\end{abstract}

Key words. infrared: stars - ISM: individual: S87 - ISM: reflection nebulae - stars: formation

\section{Introduction}

Near-infrared imaging has proved to be a valuable astrophysical tool for understanding star formation and early stellar evolution in dense molecular clouds. Because of the greatly reduced extinction in the infrared and the fact that young stellar objects emit most of their energy at wavelengths longer than $1 \mu \mathrm{m}$, near-infrared imaging enables direct explorations of deeply embedded young clusters. Stars in these clusters could reveal not only the temporal evolution of a young stellar group, but also the spatial structure of young stars, and the interactions between the cluster and its ambient gas. Our near-infrared observations toward the star forming region $\mathrm{S} 87$, also a molecular outflow source, led to the discovery of an embedded young cluster, and the elucidation of the relationships between the cluster and its ambient matter.

S87 $\left(19^{\mathrm{h}} 44^{\mathrm{m}} 13.5^{\mathrm{s}}, 24^{\circ} 28^{\prime} 00^{\prime \prime}, 1950.0\right)$ is an active site of massive star formation at a distance of $2.1 \mathrm{kpc}$ (Clemens 1985). This region has been observed in the optical (Sharpless 1959; Barsony 1989), far-infrared (Price \& Walker 1976), nearinfrared (see Barsony 1989), and radio continuum (Felli \& Harten 1981; Bally \& Predmore 1983; Barsony 1989). The S87 HII region is embedded in a $3.6 \mathrm{pc}$ diameter molecular cloud of

Send offprint requests to: Xing-Wu Zheng,

e-mail: xwzheng@nju.edu.cn roughly $7000 M_{\odot}$. The $\mathrm{CO}$ observations show that a core $\sim 2^{\prime}$ $(1.2 \mathrm{pc}$ for a distance of $2.1 \mathrm{kpc})$ in diameter and $500-1000 M_{\odot}$ in mass is located in the center of the cloud (Bally 1983). VLA maps with arcsecond angular resolution of the HII region S87 show that it has a cometary shape with a head approximately 0 '.73 $\times 0$ ' 67 in size and a $\sim 30^{\prime \prime}$ extended, low-intensity emission tail, trailing to the southeast. Radio continuum observations suggest a Lyman continuum flux of $1.9 \times 10^{47}$ photons $^{-1}$, corresponding to an embedded B0 ZAMS (Barsony 1989). A northeast-southwest oriented molecular outflow had been carefully mapped with high spatial resolution CS $(J=2 \rightarrow 1)$ and ${ }^{13} \mathrm{CO}(J=1 \rightarrow 0)$ observations. The high velocity ${ }^{13} \mathrm{CO}$ and $\mathrm{CS}$ emission lobes are spatially coincident. From the spatial and velocity structure of the molecular gas Barsony suggested an outflow geometry with an inclination angle $i=50^{\circ}$, cone opening angle $q=60^{\circ}$, and ratio of maximum/minimum cone radius is 10 . These phenomena demonstrate the site to be an active star formation region with the attendant bipolar molecular outflow.

We have obtained near-infrared images of the $\mathrm{S} 87$ region in the $J H K^{\prime}$ broad bands and the $\mathrm{H}_{2} v=1-0 \mathrm{~S}(1)$ narrow band. Our observations reveal a cluster of obscured stars with infrared nebulosity in the outflow region. The near-infrared data allow us to investigate cluster properties and nebular structure and provide insights into the relationship between the 


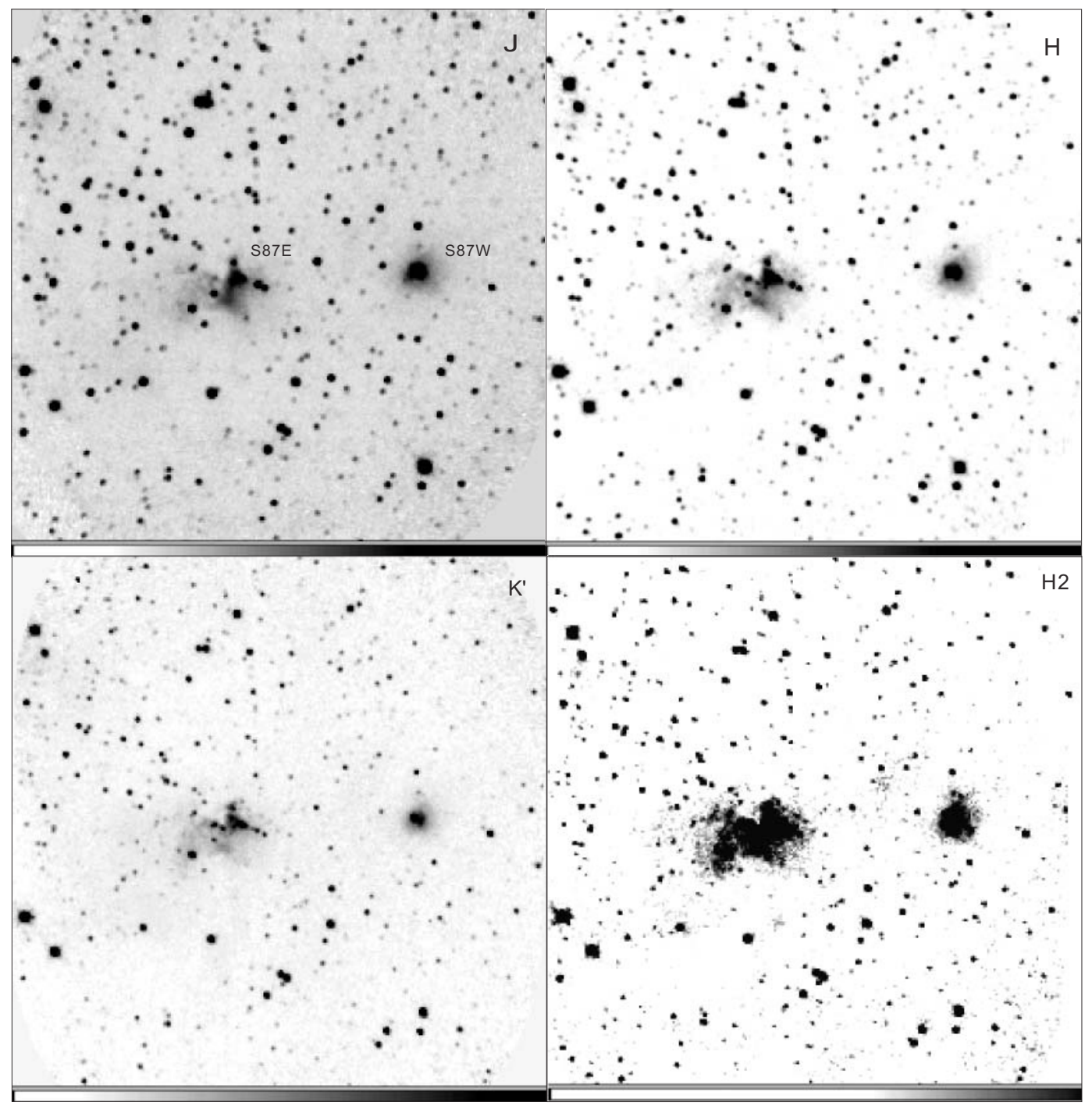

Fig. 1. $J, H, K^{\prime}$ and $\mathrm{H}_{2} v=1-0 \mathrm{~S}(1)$ images of the active star formation region of S87 over the $4^{\prime} \times 4^{\prime}$ area. Upward is north and the left is east. There are two nebulae in the region, labelled S87E and $\mathrm{S} 87 \mathrm{~W}$ in this paper.

molecular outflow, the infrared nebulosity, and the $\mathrm{H}_{2}$ line emission. This paper is organized into five sections. Section 2 briefly describes the observations and data reduction. Section 3 shows the results. Section 4 discusses the implications of our results, and in Sect. 5 we summarize our conclusions.

\section{Observation and data reduction}

The observations were carried out on October 21, 1997 at the $1.88 \mathrm{~m}$ telescope in Okayama Astronomical Observatory, Japan. The infrared camera, OASIS (Yamashita et al. 1995; Okumura et al. 2000), equipped with a NICMOS3 array, provides a field of view of $4.2 \times 4.2$ arcmin $^{2}$ with a plate scale of 0.97 pixel $^{-1}$. Five dithered $H, K^{\prime}(2.12 \mu \mathrm{m})$ images and six $J$ images were obtained for the $\mathrm{S} 87$ region. The total integration times were 120,50 , and $25 \mathrm{~s}$, respectively, for the $J, H$, and $K^{\prime}$ bands. During the entire observation period the weather conditions were excellent. The $F W H M$ of the seeing disk was measured to be 1"'8 2'. 0 . The observations of $\mathrm{H}_{2} v=1-0 \mathrm{~S}(1)$ $(2.16 \mu \mathrm{m})$ were carried out on October 30,1999 , and $K^{\prime}$ images were also obtained during the observation.
The images were processed with the IRAF packages. Every image was dark-subtracted, flat-fielded, and sky-subtracted. The flat field was obtained by two sets of dome flat frames taken with a lamp on and off. The background sky frames were obtained by median-filtering the flat-fielded data frames. The reduced images were registered and aligned using common stars and then median combined. The astrometry was conducted to obtain the coordinates of each pixel.

To obtain an $\mathrm{H}_{2}$ emission-line image, a continuum image should be subtracted from the observed $\mathrm{H}_{2}$ image. The weighted $K^{\prime}$ image taken on the same night was employed as the continuum image. Some field stars, believed to have no $\mathrm{H}_{2}$ line emission, were chosen for getting the $\mathrm{H}_{2} / K^{\prime}$ flux ratios. The median of these ratios was taken as the weighting factor.

\section{Results}

Figure 1 presents the $J, H, K^{\prime}$, and $\mathrm{H}_{2}$ images of the $\mathrm{S} 87$ region, covering a $4^{\prime} \times 4^{\prime}$ area, corresponding to $2.4 \mathrm{pc} \times 2.4 \mathrm{pc}$ at the source. The near-infrared images reveal two bright nebulae, labelled S87E and S87W, in the dense star field. 


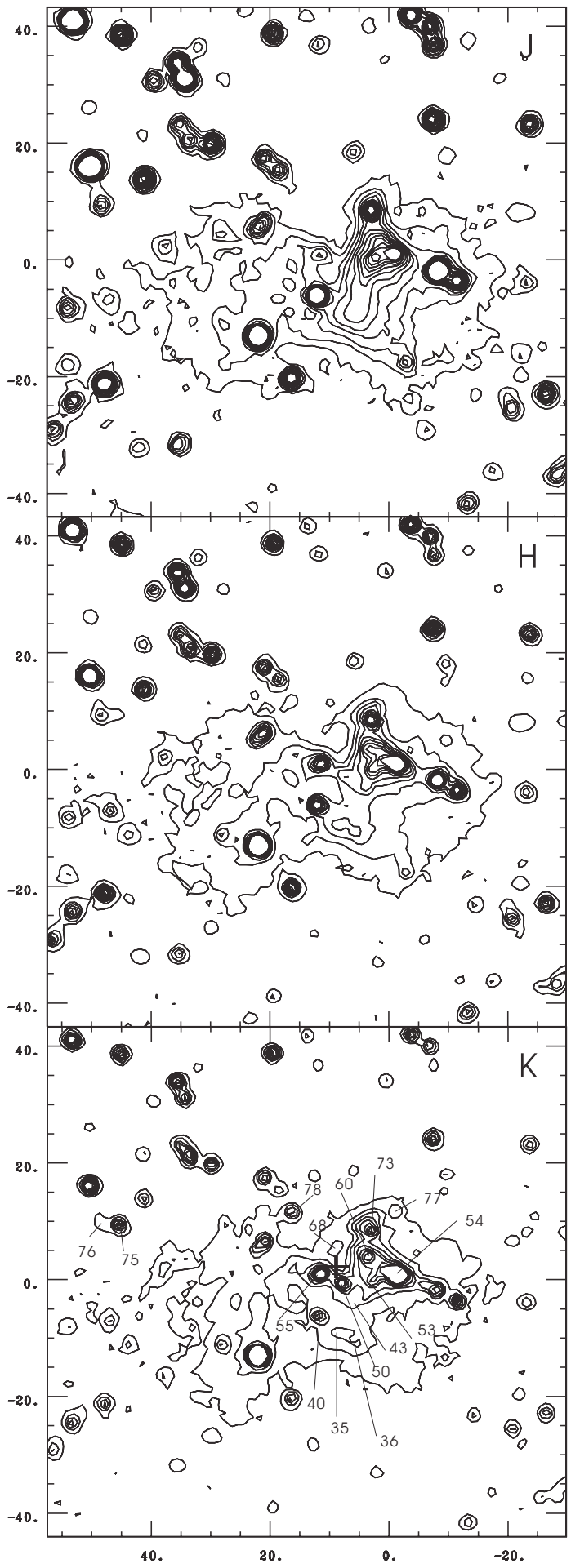

Fig. 2. Contour maps in the $90^{\prime \prime} \times 90^{\prime \prime}$ region around S87E. Coordinate axes are labelled in arcsec offsets from the position RA (1950) = $19^{\mathrm{h}} 44^{\mathrm{m}} 13^{\mathrm{s}} \cdot 1$, Dec $(1950)=24^{\circ} 28^{\prime} 03^{\prime \prime}$. The radio peak is indicated by the plus $(+)$ and some interesting infrared sources (see text) are marked by the numbers.

S87E is an M-shaped nebula associated with IRAS $19442+$ $2427\left(19^{\mathrm{h}} 44^{\mathrm{m}} 13.5^{\mathrm{s}}+24^{\circ} 28^{\prime} 00^{\prime \prime}\right)$. The symmetry axis, passing through the M-shaped nebula, is in the direction from southwest to northeast. Situated $\sim 1 .^{\prime} 5$ to the west is the S87W nebula, with a fairly round shape. Compared with the previous optical observations (Barsony 1989), our images show that in the $\mathrm{S} 87$ region, there are more point sources detected in the near-infrared emission bands than in the optical.

Contour maps of S87E in the $J, H, K^{\prime}$ bands are shown in Fig. 2. The maps are plotted with coordinates offset from the astrometry origin $\left(\mathrm{RA}=19^{\mathrm{h}} 44^{\mathrm{m}} 13.1^{\mathrm{s}}\right.$, Dec $=24^{\circ} 28^{\prime} 03^{\prime \prime}$; 1950.0). Contour map at each band clearly shows the M-shaped structure mentioned above. An opaque patch to the northeast of the nebula is particularly striking.

Sources were identified and counted using the DAOFIND routine of IRAF package, using a $F W H M$ of PSF around 2.5 pixels and detection threshold equal to four sigma. The $J H K^{\prime}$ photometry was carried out for the point sources, using the DAOPHOT package. A PSF fitting procedure was performed for all sources on each image. After removing fake stars at the edge of the field, we have extracted 501, 522, and 422 sources from the $J, H$, and $K^{\prime}$ band images, respectively, in the whole $4.2 \operatorname{arcmin}^{2}$ region. The limiting magnitudes are 18.0, 16.5, and 15.2 mag respectively for these three bands, with the uncertainty of $0.2 \mathrm{mag}$. 111 point sources were detected in the $90^{\prime \prime} \times 90^{\prime \prime}$ area around the nebula S87E (the same area shown in Fig. 2). In the contour map of the $K^{\prime}$ band, some sources associated with the nebula are labelled by the numbers, which depict the identified stars in Table 1. These sources will be discussed below. The position of the radio peak (Barsony 1989) is also marked in the contour map with a plus, locating near the edge of the opaque patch.

The $\left[H-K^{\prime}\right]-[J-H]$ diagram for these sources is plotted in Fig. 3. Solid curve represents the color-color relation for main-sequence stars, while the short-dashed line separates qualitatively the sources with IR excesses from the reddened main-sequence stars via the reddening belt by the uncertainty in observed $\left[H-K^{\prime}\right]$ colors of 0.4 mag. Only 56 sources are plotted in Fig. 3, because other sources were not detected in one or two bands in our photometric process, lacking either $\left[H-K^{\prime}\right]$ and/or $[J-H]$ values. The fact that some sources were not detected in the $J$-band, but appeared in the $H$ and $K^{\prime}$ bands, may imply that these sources are embedded much more deeply inside the molecular cloud.

In Fig. 4a, we present the $K^{\prime}$ luminosity functions for stars inside the $90^{\prime \prime} \times 90^{\prime \prime}$ area around S87E and the expected field stars. We construct the field star KLF using the stars outside the S87E region. The field KLF was scaled by the ratio of the S87E area and the field star area. We notice that the treatment may overestimate the number of field stars. However, we checked the field star KLF and found that it is very similar to the background field star KLF in NGC 1333 (Lada et al. 1996). The differential KLF of the S87E region is shown in Fig. 4b. As seen in NGC 1333, the differential KLF rises slowly with magnitude and decreases for stars with $m_{K^{\prime}}>14 \mathrm{mag}$. Here for S87E the turnover after 14th mag appears to be real and is not caused by our limit magnitude of $15.2 \mathrm{mag}$.

Figure 5 presents the $\left[J-K^{\prime}\right]$ color of the S87E region, in which the nebula shows different colors in the eastern and western area. The reddest color is seen around the opaque patch mentioned above, while the bluest color is seen in the western part of S87E with an elongation in the northeast-southwest 


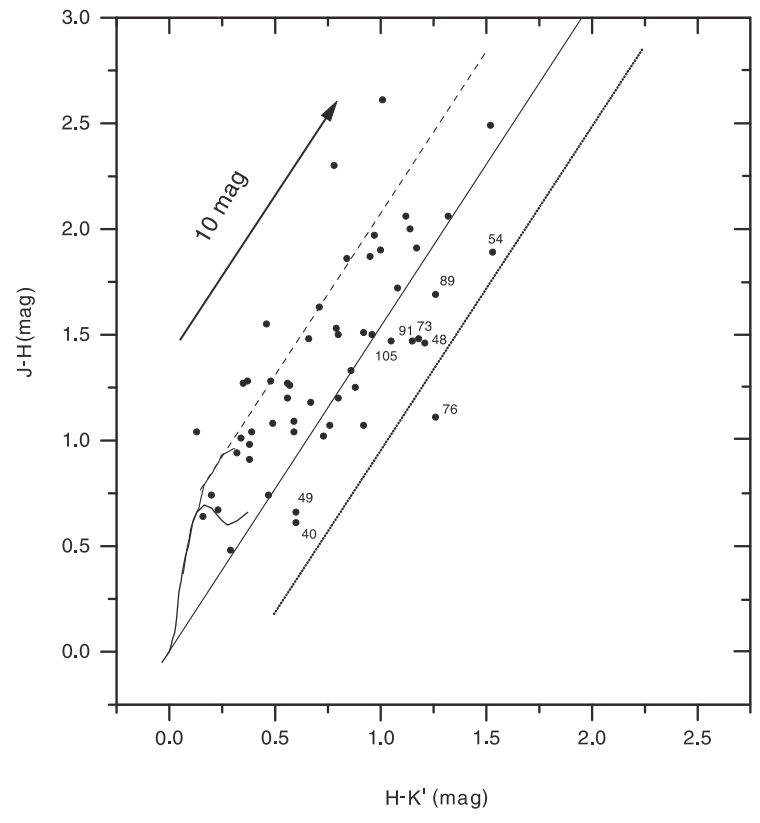

Fig. 3. $[J-H]-\left[H-K^{\prime}\right]$ color-color diagram for point sources detected in the $90^{\prime \prime} \times 90^{\prime \prime}$ area around S87E. Solid curve represents the colorcolor relation for main-sequence and giant branches stars (Bessell \& Brett 1988). The dotted line separates qualitatively the sources with infrared excesses from the reddened main-sequence stars via the reddening belt by an uncertainty in observed $\left[H-K^{\prime}\right]$ colors of 0.4 mag. A 10 mag of visual extinction vector is also plotted for reference.

direction. If the bluer colors imply less extinction, this elongation could suggest a cavity structure.

In Fig. 6, the narrow-band $\mathrm{H}_{2}$ (-continuum) emission is presented. In the image we see five $\mathrm{H}_{2}$ emission regions in the S87E and S87W fields, three (labelled as A, B, and C) of which are associated with the S87E nebula and two (D and E) with the S87W nebula. The approximate area of the HII region is also shown in the figure by a dashed-line circle.

Figure 7 presents the contour map of the S87W nebula. In this region the surface density of point sources is less than that in the S87E region. The shape of the nebula is also more regular than that of the S87E nebula.

\section{Analysis and discussion}

\subsection{Near-infrared point sources in the S87E region}

By photometry, 111 point sources have been extracted from the $90^{\prime \prime} \times 90^{\prime \prime}$ area around the S87E nebula. Most of them were not detected by previous optical observations. In our observations, only 56 of 111 point sources have been detected in all three bands. For these, we have the $[J-H]-\left[H-K^{\prime}\right]$ plot shown in Fig. 3. Such color-color diagrams can be very useful for understanding the nature of the stars in the cluster (Lada \& Adams 1992; Lada et al. 1994). Our observational data show that about 30 sources have their colors consistent with main sequence stars, which are reddened by normal interstellar extinction up to $15 \mathrm{mag}$. A total of 15 or $27 \%$ of all the $J H K^{\prime}$ point sources in the area are found in the infrared excess region which is located to the right of the reddening
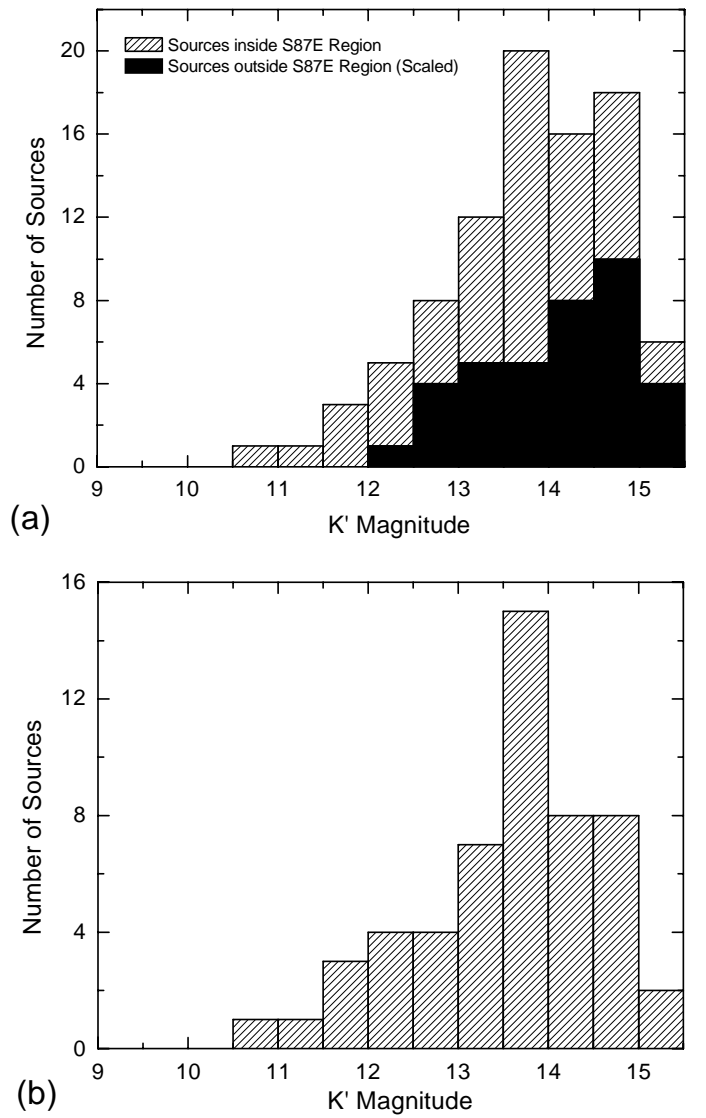

Fig. 4. a) The $K^{\prime}$ luminosity function for S87E cluster along with an appropriately scaled field star KLF. b) The differential $K^{\prime}$ luminosity function of the S87E cluster.

band. After adjusting background contamination, the fraction of near infrared excesses is expected to enhance to $40 \%$. In the $90^{\prime \prime} \times 90^{\prime \prime}$ area, 33 of 111 point sources are not detected in the $J$-band, suggesting that they are very likely young stars embedded more deeply in the molecular cloud. This suggests that the estimated fraction of infrared excess sources is possibly a low limit. Our result is very close to the fraction found within the Taurus (50\%) (Kenyon \& Hartmann 1995) and NGC 1333 cluster (60\%) (Lada et al. 1996) and nearly twice that within the IC 348 cluster (20\%) (Lada \& Lada 1995). The relatively large fraction of infrared sources in S87E indicates that this cluster is very young, between $3 \sim 4 \times 10^{6}$ years. Additional evidence for a young age of S87E is the outflow activity near the cluster associated with the earliest stage of stellar evolution.

The overall shape and structure of the luminosity function for S87E appears somewhat different from that for NGC 1333. Although both rise slowly with magnitude, the differential KLF for the double cluster in NGC 1333 is relatively flat in appearance and shows a sharp decrease for stars fainter than 14 mag. Unlike NGC 1333 there is a prominent peak in the S87E cluster at 13.5 mag. Both S87E and NGC 1333 have a consistent turnover at $m_{K^{\prime}}=14 \mathrm{mag}$, and there are still more cluster members with luminosities less than 14th magnitude at $K^{\prime}$ band.

Interestingly, 12 candidates for young stellar objects, 4 with IR excesses (NIRS 40, 49, 54, 73) and 8 without detected $J$-band emissions (NIRS 43, 50, 53, 60, 68, 72, 77, 78) are 
detected near the cone-shaped opaque patch. These 12 sources, which are closely grouped and located within a $\sim 20^{\prime \prime}$ area around the observed radio peak, form a subcluster. The reddest source in the S87E region, NIRS 54, which is located in the region typical of protostars in the color-color diagram, (Class I luminous sources, $\left[H-K^{\prime}\right]>1.5 \mathrm{mag}$ and $[J-H]>0.7 \mathrm{mag}$ ), is one member of the cluster. In the same region of the optical image (Barsony 1989), only two bright knots A and B are found. Knot A is located to the south of NIRS 53, which is most probably a piece of nebulosity reflecting the light of NIRS 53, while knot B coincides spatially with NIRS 54 and may be the optical counterpart of the infrared source.

The site of the opaque patch must be denser than the other part of the nebulae, so it is not surprising that the infrared subcluster lies near it. The HII region is found beside the subcluster and extends to the east. The $\mathrm{H}_{2}$ emissions discussed below found surrounding the subcluster. The radio peak also lies inside the subcluster. All these clues suggest that active star formation is on-going in the subcluster. Either the subcluster or the radio peak could be responsible for the $\mathrm{HII}$ region and the $\mathrm{H}_{2}$ emission.

\subsection{The infrared nebulosity of S87E}

Comparing our near-infrared images with the optical image of the S87 region, we perceived that the near-infrared observations provide further insight into the nebular structure. In the optical image, only two bright knots are seen in the region, while in near-infrared images many more point sources, apart from the two bright nebulae, were detected in the region. Overall, the S87E nebula is much brighter and redder than the S87W nebula. More near-IR sources are associated with the S87E nebula than with the S87W nebula. This implies that there are more dust and gas in the eastern part of the region than in the western part.

As mentioned above, in the $\left[J-K^{\prime}\right]$ color map (Fig. 5), the reddest color is seen around the opaque patch, while the bluest color shows a cavity in the western part. Our near-IR images reveal that the angle of the cone-shaped opaque patch is $\sim 65^{\circ}$ and the axis inclined at a position angle (p.a.) $\sim 45^{\circ}$. These are approximately consistent with the outflow geometry proposed by Barsony (1989). To investigate the connection between the molecular (CO) outflow from the young star and the opaque patch and the cavity, we outline the CO outflows in Fig. 5, where solid line stands for the blue-shifted lobe and dashed line shows the red lobe. The outflow axis is approximately consistent with the symmetry axes of the M-shaped nebula, the coneshaped opaque patch and the cavity. From the map one can find that the cone-shaped opaque patch corresponds to the red lobe of the outflow. This is probably because the outflow goes away from the nebula and leaves dust behind. The color map also shows that the blue cavity is spatially coincident with the blue lobe of the outflow, which indicates that the cavity is formed by the blue lobe of outflow approaching the observer and sweeping away the materials in the molecular cloud. It is also true that in this cavity, young stars seldom were found because of insufficient material from which to form. From previous VLA continuum observations (Barsony 1989), the HII region is located in the eastern part of S87E. The western part of S87E, including the blue cavity, is likely to be a reflection nebula. From the eastern part to the western part in S87E, the radio HII region, the infrared cluster and the molecular outflow reveal a sequence of progressively younger objects indicating the different stages of evolution.

\subsection{The $\mathrm{H}_{2}$ emission}

In Fig. 6, the $\mathrm{H}_{2}$ emission shows a rather complex structure. Due to the low signal-noise ratio, we can only roughly describe some of the characteristics of the $\mathrm{H}_{2}$ emission. In the figure, the HII region is also outlined by a dashed-line circle.

There are five $\mathrm{H}_{2}$ regions discovered in the whole $\mathrm{S} 87$ field, of which regions A, B, and C are associated with S87E, while D and $\mathrm{E}$ seem to be associated with $\mathrm{S} 87 \mathrm{~W}$. Region A, the largest and most complex region, lies in almost the same area as S87E. We can see that the eastern part of region A lies inside the HII region, which suggests that they could be related. The western part of region A surrounds the young cluster mentioned above. Region B contains two bow-shaped knots, B1 and B2, and some diffuse emissions. The two knots, B1 and B2, surrounding the HII region, may be associated with the HII region. Region $\mathrm{C}$ appears to be an elongation from the S87E nebula, extending about 100 arcsec long, or 1 pc. This $\mathrm{H}_{2}$ region maybe suggests a jet from the S87E nebula. Region D surrounds NIRS A and NIRS B inside S87W area and are probably excited by the stars. Region $\mathrm{E}$ is a bow-shaped knot extending 70 arcsec long, 40 arcsec away from the S87W nebula, could be due to shocks driven by the stars inside S87W.

\section{4. $S 87 W$}

In contrast to the $\mathrm{S} 87 \mathrm{E}$, the structure of $\mathrm{S} 87 \mathrm{w}$ nebula is quite simple. About 30 sources were detected in the $\sim 50^{\prime \prime} \times 50^{\prime \prime}$ area around the bright point source NIRS A. In the $\left[J-K^{\prime}\right]$ color map (see Fig. 5), the eastern part is very blue and the red part surrounds NIRS A to its west, so the western part suffers more extinction than the eastern part. Both NIRS A and NIRS B show normal reddening according to main-sequence stars, which means they are not young stars. As discussed above, two $\mathrm{H}_{2}$ regions are also found associated with S87W.

\section{Conclusions}

We have obtained near-infrared $J, H, K^{\prime}$ images as well as an $\mathrm{H}_{2}$ emission map for the star-forming region $\mathrm{S} 87$. The main results can be summarized as follows:

Our near-infrared observations reveal the complex structure of the S87 region, in which we detected many more point sources in the near-infrared than in the optical. The eastern part of S87, S87E, is brighter and is associated with more point sources. A sharp cone-shaped opaque patch is found in the northern part of the nebula, which is probably a dense clump of gas and dust.

The $\left[J-K^{\prime}\right]$ color image shows that the western part of the S87E nebula is very blue and coincides with the optically 


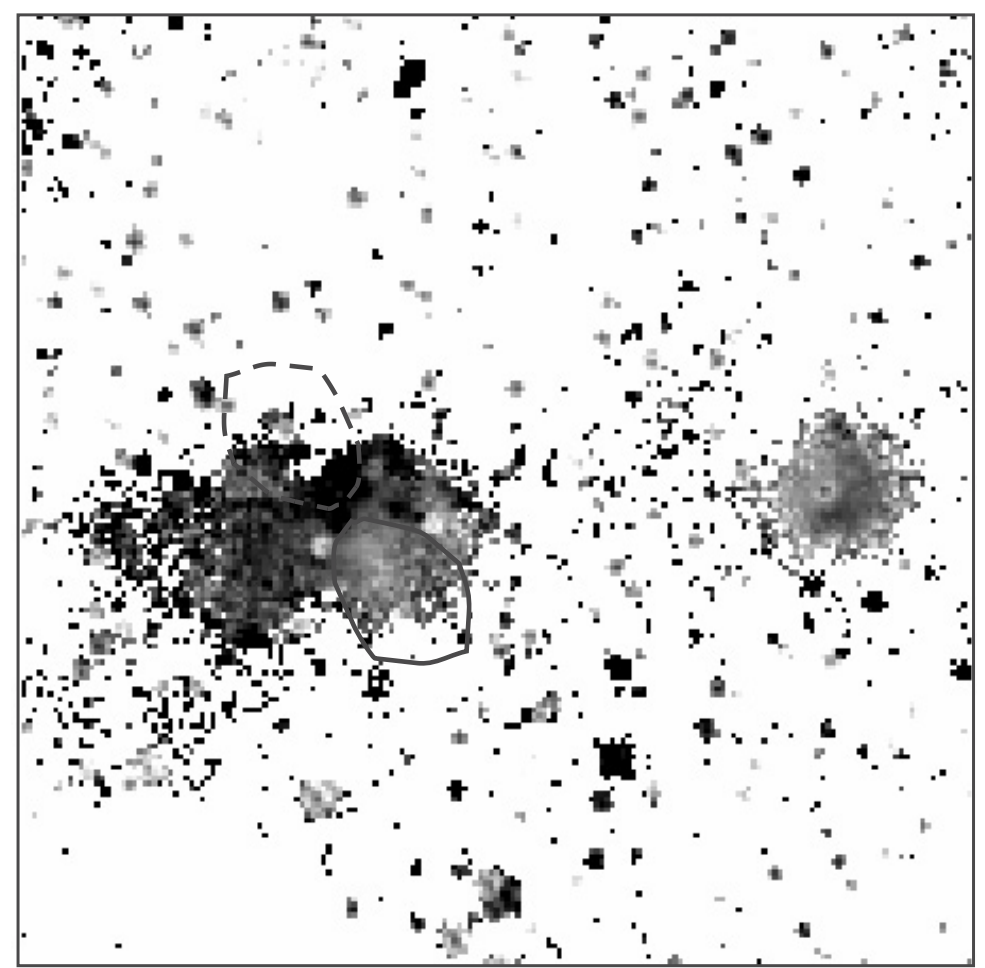

Fig. 5. $\left[J-K^{\prime}\right]$ color map over the $180 \times 180 \operatorname{arcsec}^{2}$ area around S87. The colors are linearly scaled from 0 mag (white) to 5 mag (black). The molecular outflow is outlined by the dashed line (the red-shifted lobe) and the solid line (the blue-shifted lobe).

Table 1. Photometry results of some infrared sources inside the area of S87E.

\begin{tabular}{rrrrrrrrrrr}
\hline \hline NIRS & $\begin{array}{r}\Delta \alpha \\
\left({ }^{\prime \prime}\right)\end{array}$ & $\begin{array}{r}\Delta \delta \\
\left({ }^{\prime \prime}\right)\end{array}$ & $\begin{array}{r}J \\
(\mathrm{mag})\end{array}$ & $\begin{array}{r}J \text { Error } \\
(\mathrm{mag})\end{array}$ & $\begin{array}{r}H \\
(\mathrm{mag})\end{array}$ & $\begin{array}{r}H \text { Error } \\
(\mathrm{mag})\end{array}$ & $\begin{array}{r}K^{\prime} \\
(\mathrm{mag})\end{array}$ & $\begin{array}{r}K^{\prime} \text { Error } \\
(\mathrm{mag})\end{array}$ & $\begin{array}{r}J-H \\
(\mathrm{mag})\end{array}$ & $\begin{array}{r}H-K^{\prime} \\
(\mathrm{mag})\end{array}$ \\
\hline 35 & 9.3 & -10.2 & - & - & 14.4 & 0.16 & 13.2 & 0.12 & - & 1.20 \\
36 & 6.6 & -10.2 & 15.5 & 0.12 & - & - & 13.1 & 0.13 & - & - \\
40 & 13.3 & -7.1 & 14.3 & 0.02 & 13.7 & 0.09 & 13.1 & 0.13 & 0.61 & 0.60 \\
43 & 7.8 & -5.2 & - & - & - & - & 13.7 & 0.27 & - & - \\
48 & -16.1 & -3.1 & 16.9 & 0.11 & 15.4 & 0.11 & 14.2 & 0.13 & 1.46 & 1.21 \\
49 & -6.8 & -2.8 & 13.7 & 0.01 & 13.1 & 0.03 & 12.5 & 0.04 & 0.66 & 0.60 \\
50 & 8.0 & -2.0 & - & - & 14.7 & 0.31 & 12.6 & 0.12 & - & 2.12 \\
53 & 3.5 & -0.4 & - & - & 13.1 & 0.05 & 11.8 & 0.05 & - & 1.24 \\
54 & 0.1 & -0.1 & 14.5 & 0.07 & 12.6 & 0.04 & 11.1 & 0.02 & 1.89 & 1.53 \\
55 & 12.8 & 0.0 & 16.2 & 0.27 & 13.7 & 0.10 & 12.2 & 0.08 & 2.5 & 1.50 \\
60 & 4.0 & 2.2 & - & - & 13.0 & 0.07 & 11.9 & 0.08 & - & 1.12 \\
68 & 11.2 & 6.6 & - & - & - & - & 14.0 & 0.06 & - & - \\
72 & -2.0 & 7.3 & - & - & 15.4 & 0.30 & 13.8 & 0.20 & - & 1.62 \\
73 & 4.2 & 7.5 & 14.6 & 0.01 & 13.1 & 0.02 & 11.9 & 0.03 & 1.48 & 1.18 \\
75 & 46.9 & 8.4 & - & - & 15.9 & 0.10 & 13.2 & 0.03 & - & 2.78 \\
76 & 49.6 & 8.4 & 16.1 & 0.03 & 15.0 & 0.03 & 13.7 & 0.05 & 1.11 & 1.26 \\
77 & 1.4 & 10.4 & - & - & 14.5 & 0.02 & 13.2 & 0.03 & - & 1.32 \\
78 & 18.1 & 10.4 & 16.2 & 0.04 & - & - & 13.7 & 0.09 & - & - \\
89 & 34.9 & 19.9 & 15.4 & 0.02 & 13.7 & 0.01 & 12.5 & 0.01 & 1.69 & 1.26 \\
91 & 36.6 & 22.2 & 15.6 & 0.02 & 14.1 & 0.02 & 13.0 & 0.02 & 1.47 & 1.15 \\
105 & 20.8 & 37.9 & 15.4 & 0.02 & 13.9 & 0.02 & 12.9 & 0.02 & 1.47 & 1.05 \\
\hline
\end{tabular}

visible region; the eastern and northern parts are red and coincide with the radio HII region. The asymmetric color distribution implies an asymmetric distribution of extinction. The bluest part presents an elongated cavity in the direction aligned with the blue-shifted molecular outflow. The reddest part is near the cone-shaped boundary, containing a subcluster of 12 young stellar objects and nebulae around them. From east to west in S87E, the radio HII region, the infrared cluster, and the 


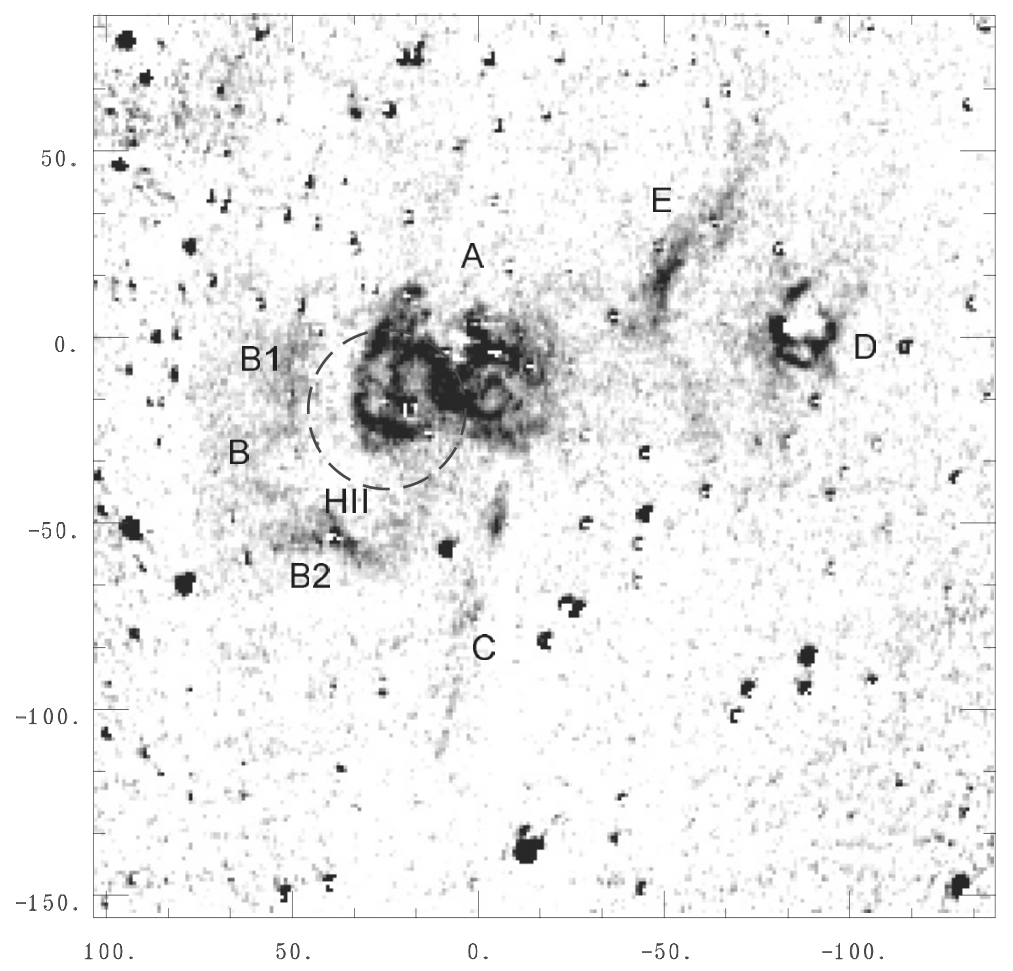

Fig. 6. The gray-scale $\mathrm{H}_{2}$ emission map of S87. Five $\mathrm{H}_{2}$ regions are marked in the figure. The HII region is also marked in the figure.

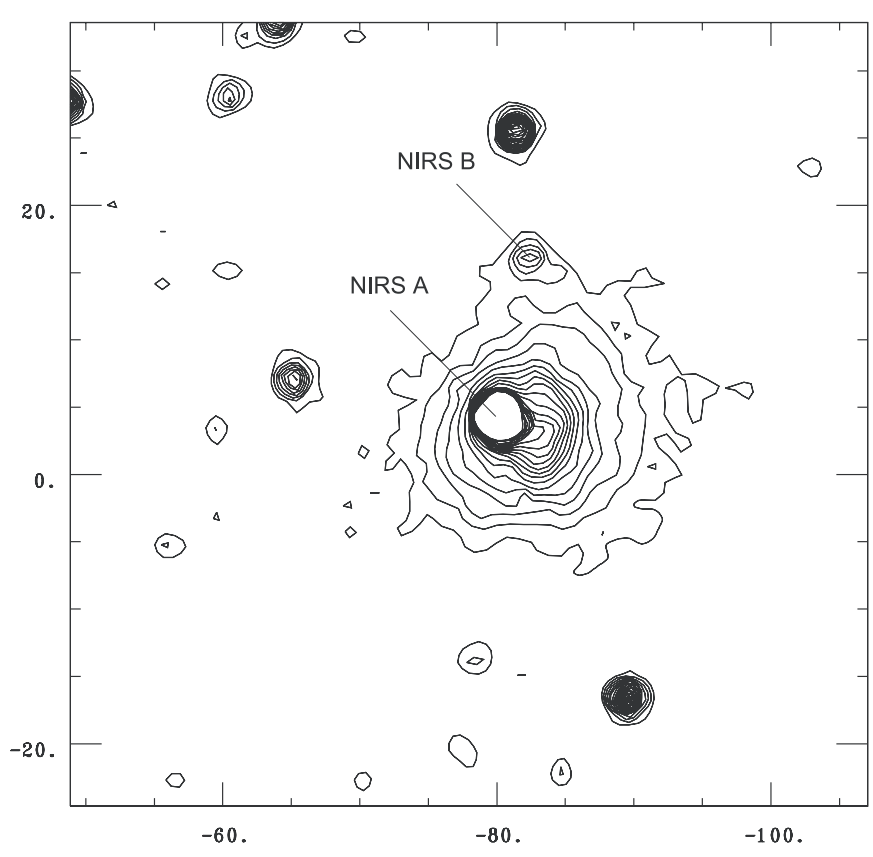

Fig. 7. $K^{\prime}$ band contour map around $\mathrm{S} 87 \mathrm{~W}$. The lowest level is $1.9 \times$ $10^{-20} \mathrm{~W} \mathrm{~cm}^{-1} \mu \mathrm{m}^{-1} \mathrm{pix}^{-1}$ and step is $2.4 \times 10^{-20} \mathrm{Wcm}^{-1} \mu \mathrm{m}^{-1} \mathrm{pix}^{-1}$. Coordinate axes are labelled in arcsec offset from the position RA $(1950)=19^{\mathrm{h}} 44^{\mathrm{m}} 13^{\mathrm{s}} \cdot 1$, Dec $(1950)=24^{\circ} 28^{\prime} 03^{\prime \prime}$.

molecular outflow reveal a sequence of progressively younger stellar objects which indicate the different stages of evolution.

According to our photometry results, we find that $40 \%$ of all sources associated with S87E exhibit infrared excesses.
With the evidence of outflow activity near the cluster, we conclude that the population within the S87E cluster is very young $\left(\sim 3-4 \times 10^{6} \mathrm{yr}\right)$

The overall shape and structure of KLF for S87E appears somewhat different from other young clusters. In the differential KLF diagram, S87E also shows a common turnover at $m_{K}=14 \mathrm{mag}$. There is a prominent peak in the S87E cluster at $13.5 \mathrm{mag}$ and still more cluster members with luminosities less than 14th magnitude at $K^{\prime}$.

Five $\mathrm{H}_{2}$ regions are discovered in the field, three of which are associated with S87E and two are associated with the S87W nebula. Among those associated with S87E, region A surrounds the whole S87E area; the bow-shaped B lies outside the HII region; and region $\mathrm{C}$ is most probably a jet from the thick $\mathrm{S} 87 \mathrm{E}$ cloud. Of the two $\mathrm{H}_{2}$ regions associated with $\mathrm{S} 87 \mathrm{~W}$, region $\mathrm{E}$ might be shocked by high speed stellar wind from the sources.

Acknowledgements. The authors would like to thank the staff of the Okayama Astrophysics Observatory for the observations. Yuxi Chen wishes to thank Dr. Paul T. P. Ho of Harvard Smithsonian Center for Astrophysics for kind help and useful suggestions to the paper. We also thank the anonymous referee for reading our manuscript carefully and giving us helpful comments. This work is supported by grants NSFC 10073004, 19803005, G19990754.

\section{References}

Bally, J., \& Predmore, R. 1983, ApJ, 265, 778

Bally, J., \& Lada, C. J. 1983, ApJ, 265, 824 
Barsony, M. 1989, ApJ, 345, 268

Bessel, M. S., \& Brett, J. M. 1988, PASP, 100, 1134

Clemens, D. P. 1985, ApJ, 295, 422

Felli, M., \& Harten, R. H. 1981, A\&A, 100, 42

Horner, D. J., Lada, E. A., \& Lada, C. J. 1997, AJ, 113, 1788

Kenyon, S. J., \& Hartmann, L. 1995, ApJS, 101, 117

Lada, C. J., \& Adams, F. 1992, ApJ, 393, 278

Lada, C. J., Lada, E. A., Clemens, D., \& Bally, J. 1994, ApJ, 429, 694

Lada, E. A., \& Lada, C. J. 1995, AJ, 109, 1682

Lada, C. J., Alves, J., \& Lada, E. 1996, AJ, 111, 1964
Shin-ichiro Okumura, Eiji Nishihara, \& Etsuji Watanabe 2000, PASJ, 52,931

Price, S. D., \& Walker, R. G. 1976, The Four-color Infrared Sky Suvey; Catalog of Observations at 4.2, 11.0, 19.8 and $27.4 \mu \mathrm{m}$ (Air Force Geophysical Lab. Tech. Rept. 76-0208)

Sharpless, S. 1959, ApJS, 4, 257

Yamashita, T., et al. 1995, in Scientific and Engineering Frontiers for 8-10 m Telescopes, ed. M. Iye, \& T. Nishimura (Tokyo: Uni. Acad.), 285 